\title{
News: Interview, Recognitions, and Societies
}

Published online: 30 May 2017

(C) The Regenerative Engineering Society 2017

\section{An Interview with David M. Gardiner}

Professor David M. Gardiner, Ph.D., is a professor of Developmental \& Cell Biology at the School of Biological Sciences, University of California, Irvine, and the Director of the regeneration lab where they investigate limb development, growth, and regeneration. Professor Gardiner also serves as an Associate Dean for Research, School of Biological Sciences, University of California, Irvine. In addition, Professor Gardiner is a Fellow of the American Association for the Advancement of Science based on his contributions in the field of regeneration research. He is an Associate Editor of Regenerative Engineering and Translational Medicine.

Question: Salamanders are the only vertebrate animals that have regenerative function for the lost body parts. Your research on the regenerative abilities of salamanders could offer valuable information to create breakthrough approaches for regenerative therapy. Which scientific aspect of this research are you most proud of?

Dr. Gardiner: I am most excited about our successes in developing and using the Accessory Limb Model (ALM) for regeneration. Historically regeneration studies have been loss-of-function (you experimentally inhibit the regenerative response and then try to rescue it). This tells you that something is important (e.g. nerves), but does not tell you how or why it is important. With the ALM (a gain-of-function assay) you can test factors for their ability to induce regeneration of an accessory (ectopic) blastema or limb, and then study what pathways you activated leading to induced regeneration. By this approach, people from the lab have identified a cocktail of growth factors that can substitute for nerve signaling, and we now can study how activation of these pathways could induce a regenerative response in a mammal.

Question: How far away would you say we are from a full regeneration of a body part?

Dr. Gardiner: That is hard to know. From the ALM we know that regeneration is step-wise and that if one of the steps is defective, then it is not possible to progress to the next step and regeneration fails. Humans must be defective in at least one of these steps. We do not know how many steps there are, or how many of them we are defective at. It seems unlikely that we have problems with them all, so the challenge is to identify the steps and fix those that are a problem. If there are not a lot of steps, and most of them work in humans, then we could be very close; obviously the opposite is also possible. The one thing we know is that it will take a finite amount of research effort, and more effort now will get us there sooner.

Question: One of your main research focuses is on understanding how blastema, the regenerating site of an amputated limb of salamander, facilitates regeneration. 
What is the most interesting finding of yours on this topic?

Dr. Gardiner: There are previous experiments demonstrating that if you let a blastema form and then graft it to an ectopic location on the animal, it will develop autonomously into a new limb. In the process of trying to reverse engineer the blastema we realized that this is only true if you also graft a bit of the stump tissues as well. Over several years, we determined that the cells of the blastema go through a stage during which they are developmentally "plastic" such that their identity and fate can be reprogrammed. This actually makes sense since the challenge of regeneration is to build new structures from the cells of the old structures that remain behind after injury. If those cells cannot be reprogrammed, then how could you make new from old? These findings have allowed us to begin investigating the underlying mechanisms for regulating the state of plasticity of blastema cells in order to control in situ reprogramming of cellular identify.

Question: What are some key questions you are still working towards finding answers with regard to blastema?

Dr. Gardiner: Our long term focus has been on the question of positional information. For successful regeneration you need regeneration-competent cells in order to rebuild the lost structures, but you also need the instructions (blueprint) for how to put the bits and pieces together. These instructions are what we refer to as positional information (PI). We know this information is made by and encoded in the extracellular matrix by cells localized in the loose connective tissues of the body. The challenge is that we do not have markers for these cells, and thus it is not possible at this time to isolate and characterize their behavior and function. We have evidence that one aspect of positional signaling involves modifications of proteoglycans, heparan sulfates (HS) in particular, and have identified a subpopulation of cells that express high levels of HS that makes them a strong candidate for the PI cells. Identifying the PI cells and learning how to control their behavior will be key to making the blastema and inducing regeneration.
Question: Which current projects in the field of regenerative engineering do you think are the most promising?

Dr. Gardiner: A major focus of our research is on how the extracellular matrix controls growth factor signaling during regeneration. This seems to me to be a great opportunity for regenerative engineering. Much of the expertise and technology is already in place to engineer a smart matrix, and the question is what needs to be engineered. Since growth factors/morphogens (e.g. FGF and BMP) are specifically bound up by sulfated proteoglycans, it makes sense to engineer the matrix in order to control endogenous signaling in a way that allows the cells to participate in regeneration, rather than to deliver non-physiological levels of exogenous factors in an attempt to tell the cells what to do.

Question: Translating research approaches for clinical use is still a huge hurdle. What do you think researchers can do to overcome this better?

Dr. Gardiner: Because we work with salamanders, the reality is that our work is a long way from translation. Nevertheless, because these animals are so good at regeneration, they provide the opportunity to understand what needs to be translated. Therefore, success comes from discovery of the important concepts and principles that can be combined with the expertise of working with a model system for translation to the clinic (e.g. rat or rabbit). This can be accomplished by finding the "right" collaborators. Collectively they need to have the right tools, but most importantly they have to share the commitment to make regeneration a reality. They need to be willing to listen and understand what each other is saying, and appreciate the value of each other's view of how it all works.

Question: What advice would you give young researches in the tissue engineering field who are starting their careers? 
Dr. Gardiner: Pick important problems, which you already have done if you are working in this area. Success in terms of advancing the field will necessitate having an open mind that will lead to coming up with lots of ideas. Evaluate your ideas critically and carefully, and choose which to investigate wisely. Remember that thinking up ideas is easy but working on them is hard.

\section{Awards and Recognitions}

Professor Ali Khademhosseini, Ph.D. (Brigham and Women's Hospital, Harvard Medical School), was honored with the 2017 Clemson Award for Contributions to the Literature from the Society for Biomaterials. This award is given to investigators who made important achievements to the literature on the science or biomaterial technology. Professor Khademhosseini has made significant contributions in the research field related to developing micro- and nanoscale biomaterials for regulating cellular behavior for tissue engineering.

Professor Samir Mitragotri, Ph.D. (University of California, Santa Barbara), received the Clemson Award for Applied Research from the Society of Biomaterials. His major research focus is drug delivery, and he has developed many innovative technologies such as skin patches based on needle-free drug delivery for treating vascular diseases and cancer. Professor Mitragotri also serves as Editor-in-Chief of Bioengineering \& Translational Medicine, an official journal of the American Institute of Chemical Engineers (AlCheE) and the Society for Biological Engineering (SBE).

Dr. Yu Shrike Zhang, Ph.D. (Brigham and Women's Hospital, Harvard Medical School), was named as an Outstanding Young Investigator from the Society of Toxicology. Since its founding in 1961, the Society of Toxicology has been organized by representative scientists in toxicology from academic institutions, industry, and government. This award has been recognizing early-career investigators who made important achievements in the field of toxicology in biotechnology. Dr. Zhang was honored for his research achievement in organs-on-chips which recapitulated the structure and functions of live human organs. Dr. Zhang was also selected as an ACS Nano Junior Fellow.

\section{Academy and Society Inductions}

Professor Nicholas Peppas (The University of Texas at Austin) was elected to the American Academy of Arts and Sciences (AAAS), based on his significant contribution in biomaterials and oral drug delivery systems. Since it was founded in 1780, the AAAS has been one the oldest learned societies and independent policy research centers in the USA, providing authoritative and nonpartisan advice to decision-makers in academia, industry, and government, through studies and programs. Professor Peppas will be inducted on October 7, at a ceremony in Cambridge, MA.

Professor Ioannis V. Yannas, Ph.D. (Massachusetts Institute of Technology), was elected as a new member of the National Academy of Engineering (NAE). This academy membership honors scientists who have made important achievements to "engineering research, practice, or education, including, where appropriate, significant contributions to the engineering literature" and to "the pioneering of new and developing fields of technology, making major advancements in traditional fields of engineering, or developing/implementing innovative approaches to engineering education." Professor Yannas was recognized for co-developing the first commercial artificial skin that saved the lives of thousands of burn patients.

\section{University Positions}

Professor Gordana Vunjak-Novakovic, Ph.D. (Columbia University), was appointed as University Professor for the first time in Columbia Engineering, which is Columbia's rare and highest academic honor. She is a pioneer in the field of regenerative medicine by designing organ on a chip and engineering functional human tissues, including bone implants for facial reconstruction, electromechanically functional cardiac tissue, and donor lung recovery for transplantation.

\section{Views: Recent Advances in the Field}

\section{Advancements in Industry}

The Global Translational Regenerative Medicine market that includes stem cell therapies, gene therapies, and tissue-engineered products to treat cardiovascular diseases and cancer is anticipated to significantly grow with a faster pace in the coming years. In the year 2016, the global Translational Regenerative Medicine market was valued at 5.8 billion dollars. Visiongain, a market analysis company, forecasts the market value to increase to 14.5 billion dollars in 2021. In the first half of the forecast period, the market is expected to grow at a compound annual growth rate (CAGR) of $19.9 \%$ and then at $17.7 \%$ from 2016 to 2027 (https://www. visiongain.com/Report/1843/Global-TranslationalRegenerative-Medicine-Market-Prospects-2017-2027). 


\section{Advancements in Academia}

Scientists at California Institute of Technology have developed a new bone clearing technique (Bone CLARITY) which enabled bone to become transparent to monitor the stem cells inside, in an intact manner. Based on genetically engineered mice that have fluoresce stem cells for easy imaging, the team removed calcium from the skeletal tissues, because calcium is involved in opacity and the content of calcium in bone is much higher than soft tissues that already have established clearing techniques. The team then infused a hydrogel into the skeletal tissue to lock cellular components such as proteins for preserving original architecture. Finally, lipid of tissue was removed by using a gentle detergent, resulting the bone transparent. This technique induced a breakthrough for understanding interaction mechanism of bone with surrounding tissues, simulating bone diseases, and testing new therapeutics such as osteoporosis treatment. This research was published in Science Translational Medicine (A. Greenbaum et al. "Bone CLARITY: Clearing, imaging, and computational analysis of osteoprogenitors within intact bone marrow" Science Translational Medicine, April 2017) [1].

\section{Reference}

1. Greenbaum A, et al. Bone CLARITY: clearing, imaging, and computational analysis of osteoprogenitors within intact bone marrow. Sci Transl Med. 2017;9(387):eaah6518. doi:10.1126/scitranslmed. aah6518. http://stm.sciencemag.org/content/9/387/eaah6518 